\title{
A conceptual model for finding key factors for new product development evaluation
}

\author{
Pouya S. Moghadam \\ 596161@swansea.ac.uk| School of Management, Swansea University, Bay Campus, Fabian Way, \\ Swansea, SA1 8EN \\ Rhys G. Williams \\ r.g.williams@abertawe.ac.uk | School of Management, Swansea University \\ John Mulyata \\ john.mulyata@swansea.ac.uk| School of Management, Swansea University
}

\begin{abstract}
This paper develops a conceptual model for finding key factors for new product development (NPD) evaluation. It builds on the work of the most cited and published authors on innovation management, but transfers attention from advertising aspects and efficiency, to factors identified within the NPD process such as new product project definition, a firm's resources, organisation-product fit, and commercial entity, that would lead to success with "Information acquired" being identified as the underlying key factor. This paper presents a summary of the results of correlation coefficients calculated between the factors identified and outcome measures, derived from the leading authors' work. Further, analytic hierarchy process (AHP) was used to evaluate the results of the correlation coefficients of sub-factors, which were modified by considering the ranking of each author.
\end{abstract}

Keywords. New product development (NPD); New product project; Project Evaluation.

Cite paper as: Moghadam, P. S., Williams, R. G., Mulyata, J., (2020). A conceptual model for finding key factors for new product development evaluation, Journal of Innovation Management, www.open-jim.org, 8(4), $57-77$. 


\section{Introduction}

In recent years, innovation has become an important element of economic growth in industrialised countries and is considered as an essential component of an organisation's processes, which are needed for long-term prosperity and survival.

A key function which contributes to the innovation process is new product development (NPD) (Harris \& Trainor, 2009). Cooper \& Kleinschmidt (1987) recognised two critical issues which need to be addressed by managers of any new tangible product project when selecting the most promising new products; what determines the success or failure of new products, and what differentiates between a new product's success and failure. Although many organisations allocate a substantial amount of financial, human capital and other resources to ensuring the effectiveness of the innovation function, projects can and do still fail. Failure is not necessarily caused by a lack of commitment of managers and other employees, but, conceivably, by a lack of understanding of the key drivers that constitute the processes, assets and capabilities needed for NPD project undertaking (Pisano, 2012).

This paper draws together the work of the preeminent authors on NPD, using a quantitative approach to determine an unified index of the key factors for success. The recognition of these key factors can be regarded as the first step in developing a framework for the evaluation of NPD projects. The foundation for determining these factors is the creation of a conceptual model.

An innovative model for recognizing the key factors of NPD was developed by Cooper (1979), who argued that conceptual models aid in the determination of new product outcomes and allow for deeper recognition of factors which can influence NPD performance.

This paper frames the factors and sub-factors of NPD based on rational planning and problemsolving perspectives of research. A rational plan will determine the factors that affect the financial performance of a new product project. The problem-solving theme investigates the influence of development teams, suppliers and team leaders, on the NPD processes. This theme is based on a Japanese development discipline of problem-solving which has at its core a cognitive theoretical orientation, and embraces flexible and different problem-solving methods first applied in Japanese project teams (Song \& Noh, 2006).

These two streams of rational planning and problem-solving research complement the novel theoretical framework that this paper's conceptual model uses, which is a "resource based view" (RBV) of organisations. RBV aims at determining the influence of a firm's resources, such as, people, product development, product testing and launch, on the financial performance of the NPD projects. All assets, capabilities, processes, and organisational attributes such as information and knowledge are considered as resources, which are needed for the development of strategies that enable firms to be effective and efficient in their creation of a new product project (Calantone et al., 2006).

Hence, the factors and sub-factors derived from the leading authors' works and used in this paper's conceptual model encompasses the assets and capabilities utilised in the implementation of the NPD process. 
Thieme (2007) ranked the leading authors' work in the field of innovation by the number of publications across 14 leading journals on technology and innovation management, marketing, and management, between 1990 and 2004.

This paper's conceptual model focuses on work of the leading authors recognised by Thieme, and was derived by evaluating critically the work of Robert Cooper and Elko Kleinschmidt (ranked by Thieme as 2nd and 5th respectively), Michael Song, (ranked 1st), and Roger Calantone (ranked $3 \mathrm{rd})$.

\section{Conceptual models in the literature}

Robert Cooper, in 1979, presented a conceptual model, known as project New Prod I, which was applied to the evaluation of the impact of different factors on the outcome of the new product projects. In the model, Cooper organised the factors under several categories, such as the "Nature of the project", its "Environmental category" and "Commercial entity". Cooper suggested that the success or failure of a new product project is based directly on the relationship of its "Commercial entity" with the market; other categories largely influence the commercial entity directly, therefore affecting the overall outcome indirectly. (Cooper, 1979)

Song and Parry (1997) expanded on Cooper's project New Prod I conceptual model by creating eight hypotheses that considered the direct and indirect relationships of the factors underlying Cooper's three categories:

H1 The positive effect of competitive potency level on "Market and competitor intelligence" but negatively affects "New product competitive advantages" and "Degree of new product successful out-come".

H2 The positive affect of firm's marketing skills and resources on "Market and competitive intelligence" and "Proficiency of marketing activities".

H3 The positive impact of firm's technical skills and resources on "Market and competitive intelligence" and "Proficiency of technical activities".

H4 The positive effect of cross-functional integration on "Market and competition intelligence', "Marketing activity proficiency", "Technical activity proficiency" and "New product success".

H5 The positive affect of marketing and competition intelligence on "Market activity proficiency", "Technical activity proficiency", and "New product success".

H6 Market activity proficiency positively affects "Product competitive advantage".

H7 Technical activity proficiency positively affects "Market activity proficiency" and "Product competitive advantage".

H8 Positive affect of the product competitive advantage on "The new product success".

Cooper and Kleinschmidt's (1987) conceptual model, was based on Cooper's previous research and empirical evidence namely project New Prod I and demonstrated the relationship of the environmental factors with the new product strategies and implementation. They defined new 
product strategies as the new product itself, namely, product design, product advantages, and the launch of the new product into the market. The product strategy is the outcome of the new product process from the idea generation to product launch. The relationship between environmental factors and new product strategy is that the latter is executed within the organisation's environment.

Song et al (2003) proposed a conceptual model, which examines the relationship of project management characteristics, consisting of factors such as project management style, project manager skills and senior management support, with structural and process dimensions that has factors such as "Cross-functional integration" and "Planning proficiency".

Calantone et al (2008) present a conceptual model which combines the key factors of NPD recognised in Cooper and Kleinschmidt's and Song et al. publications, by determining the direct and indirect impact of proactive strategic orientation of an organisation and organisational structure on the successful outcome of the NPD process. Calantone et al (2008) explain the proactive strategic orientation of an organisation as the aggressive action and strategies of an organisation, and the organisational structure as the work allocation and administrative aspects that directs activities, resources and coordination of these aspects of the NPD process. They also considered environmental turbulence such as the market, competition and technological changes as a moderating factor for the relationship of the inter-organisational aspects that is not under managerial control. The impact that environmental turbulence has on the relationship of the factors may influence the outcome of the NPD project.

\section{Methods of data collection and analysis used in literature}

This paper presents a new conceptual model based on an analysis of the factors and sub-factors derived by the leading authors from empirical data. The authors evaluated their conceptual models in a variety industries in different regions, and applied different statistical methods to analyse the correlation coefficient, represented as " $\mathrm{r}$ " in this paper, between the factors they derived and performance measures, categorised as financial, market and window of opportunity performance outcomes.

The aspects of new product projects which affect success, and whether there are different dimensions of success, were presented in "New Prod II" by Cooper and Kleinschmidt (1987). They investigated the extent to which the success of a new product project can be measured, and the perspectives by which it can be measured, and whether such independent measurements exist, and; what the different aspects of identifying the success of the new product project are, and whether there are similarities between them.

Cooper and Kleinschmidt (1987) gathered 200 new product project cases from 125 manufacturing organisations. Of the 200 cases, 120 were successful projects and 80 not. Managers and project leaders assigned to these projects were questioned on the nature of the product and the advantages that it offers; the product's target market and the attractiveness of the market; the organisation's commitment to the new product project and how well defined the protocols for engaging for its development were; the risk related to the purchase of the product by the con- 
sumer; and the product organisation fit or synergy, which they considered as the new product project fit with the firm's resources, skills and experience.

Cooper and Kleinschmidt's 1987 study developed on Cooper's 1979 work which examined the factors leading to the success or failure of new products by collecting data relating to the 77 factors of the conceptual model from 177 manufacturing companies from a variety of industries across Canada.

Song and Parry (1997), undertook analogous studies on 611 Japanese companies and received 788 completed questionnaires from 404 of the companies.

Song et al's 2003 study focused on data collected on 192 new product projects from Japanese and Korean OEMs, producing for example, semiconductors, electronics, computer, instrumental, audio-visual and communication equipment.

Similarly, Calantone et al's in 2008 study was conducted on 346 manufacturing organisations from the Fortune 500 global list.

Each of these studies applied a scale of $0-10$ for respondants to rate their opinions and therefore it was not necessary to rescale to a common range for the purpose of the analysis in this paper.

\section{Observations on the Literature}

Cooper's "Environmental category" was divided into three factors; "The market", "The firm's resources" and "The nature of the project". Cooper concluded that the category did not have a major impact on the outcome of the projects, contradicting the importance that was initially put on this category in his conceptual model. Most other screening models also believed environment to be an influence, however Cooper's conclusion was that it is not a significant factor.

However, a "Firm's resources" had the most impact on the outcome of the new product projects. Four of the most influential sub-factors were; "Sales force and resources", and, "Skills distribution", "The personnel and skills of the marketing research division", "Skill of management", and, "The resources and skills of the promotion and advertisement division" (Cooper, 1979).

"Proficiencies of processes" according to Cooper's analysis is the most important and noteworthy major factor affecting the outcome of the three other controllable variables. The three most significant sub-factors of this factor were "Market launch", "Prototyping with consumer input" and "Test marketing-trial selling". Therefore, it could be said that the most influential group of sub-factors that influence the success or failure is the marketing elements of the new product project process. These marketing elements were further supported by an "Information acquired" factor whose three most influential sub-factors were "Knowledge of consumer price sensitivity", "Understanding the buyer's behaviour and knowledge of consumer needs and wants", and "The technical aspects needed for the new product". From these most influential sub-factors, it could be concluded that having greater knowledge of the consumer based on their price preferences, behaviour, wants and needs, have, according to Cooper's findings, a significant impact on the success or failure of the new product project. (Cooper, 1979)

Song and Parry's (1997) work examined the case of new product selection; evaluating competitive 
environment, marketing and technical synergies are important. Furthermore, "Cross-functional integration" is highly important for the successful development of the new product project and its overall success in the market. They confirm Cooper's (1979) study that marketing and competitive intelligence, market activity proficiency, and product competitive advantage, have the greatest impact on the project management aspect of the new product project programme. However, in Japanese firms the market activity proficiency, which directly affects new product competitive advantage and new product success positively, appears to be less important.

Cooper \& Kleinschmidt's (1987) paper shows that when product cases went through the 10 performance measures, the three most critical dimensions effecting the outcome and performance of the new product project were: Financial performance, Opportunity window and Market impacts. Furthermore, according to them, some of the new product project characteristics were not affecting the any of the three performance measures, these were low price, financial synergy in terms of resources, market size, purchasing nature by the consumer and new product design. (Cooper \& Kleinschmidt, 1987)

Conversely, Song and other's 2003 study, examined three sub-factors of the planning which contributes to the survival of the new product project within the market, namely "Detailed planning", "All team members contributing to the planning process" and "Autonomy and flexibility of the project teams". "Detailed planning" could increase the efficiency of the development process that in turn could impact the efficiency and overall performance of the new product within the market. (Song et. al, 2003)

However, Calantone et al. observed that environmental turbulence, which consists of technological uncertainty and market turbulence, acts as a control for their model. They assessed that only a moderate relationship exists between innovativeness and market intelligence on the outcome of the new product project. Hence, in a low-turbulence environment market intelligence had a more positive impact on the successful outcome than the relationship between innovativeness and success which occurs during highly turbulent environments. Furthermore, a positive relationship between proactive strategy with innovativeness and marketing intelligence has been observed by their model. (Calantone et.al 2008)

This paper examined the leading authors' publications who determined the most influential factors and sub-factors with regards to NPD success, rather than the specific factors or subfactors influencing success.

Their publications correspond in terms of the similarity of their conceptual models, in the way they were validated, in the results they observed, and in terms of the organisation of the factors and sub-factors and the relationships of the factors to the successful outcome of NPD projects. However, there are minor differences in how the factors are stated, in including additional subfactors, and in their opinions on the influence of some sub-factors.

A quantitative method is used for testing and evaluating the commonalities in the five predominant NPD publications of these leading authors, and the results are summarised in table 1. 


\section{Summary table and analysis}

Table 1 summarises the results obtained from the analysis of the reviewed authors' empirical data.It includes thecorrelation coefficient $(\mathrm{r})$ between the factors derived from these authors, and performance measures, categorised under financial, market and window of opportunity performance outcomes.

The factors and sub-factors have been matched to the RBV theoretical framework and Cooper's 1979 categorisation of the key factors. In addition, the "firm's strategy" factor, in the sense used by Calantone et. al, (2008) has also been included.

The table exhibits an analytic hierarchy process (AHP) that comprises of the correlation coefficients of sub-factors (shown in Table 1 as $\mathrm{r}$ ) adjusted by the ranking of the authors in accord with Jeff Thiems' 2007 study. The table also illustrates the mean of AHP results for each key factor. The formula that the AHP used is shown in Equation 1:

$$
W K_{i}=\frac{\sum_{j=1}^{n} R_{j} K_{i j}}{\sum_{j=1}^{n} R_{j}} \quad \text { (Equation 1) }
$$

Whereas Weighted Key sub-factors (WK) represents the correlation coefficients of sub-factors adjusted by the ranking of the authors, R, on a scale of 1 to 10. Finally, K represents the correlation coefficients of each sub-factor.

Table 1. Summary table of authors results with AHP

\begin{tabular}{|c|c|c|c|c|c|c|c|}
\hline Key factors & Key sub-factors & $\begin{array}{l}\text { Cooper } \\
1979\end{array}$ & $\begin{array}{l}\text { Cooper } \\
\& \\
\text { Kleins- } \\
\text { chmidt } \\
1987\end{array}$ & $\begin{array}{c}\text { Song \& } \\
\text { Parry } \\
1997\end{array}$ & $\begin{array}{c}\text { Song et. } \\
\text { al, } \\
2003^{*}\end{array}$ & $\begin{array}{l}\text { Calantone } \\
\text { et. al, } \\
2008^{* *}\end{array}$ & $\begin{array}{l}\text { AHP } \\
\text { scoring }\end{array}$ \\
\hline \multirow{4}{*}{$\begin{array}{l}\text { Market en- } \\
\text { vironment } \\
(\text { Mean }= \\
0.09)\end{array}$} & $\begin{array}{l}\text { 1. Customer need of } \\
\text { the specific product }\end{array}$ & $\begin{array}{l}\mathrm{r}= \\
0.329\end{array}$ & $\begin{array}{l}\mathrm{r}= \\
0.313\end{array}$ & $\mathrm{r}=0.01$ & $\mathrm{~N} / \mathrm{A}$ & $\mathrm{N} / \mathrm{A}$ & 0.13 \\
\hline & $\begin{array}{l}\text { 2. Market growth } \\
\text { rate }\end{array}$ & $\begin{array}{l}\mathrm{r}= \\
0.221\end{array}$ & $\mathrm{~N} / \mathrm{A}$ & $\mathrm{r}=0.01$ & $\mathrm{~N} / \mathrm{A}$ & $\mathrm{N} / \mathrm{A}$ & 0.05 \\
\hline & $\begin{array}{l}\text { 3. Satisfaction of } \\
\text { costumers about the } \\
\text { new product } \\
\text { compared to the } \\
\text { competitors }\end{array}$ & $\begin{array}{l}\mathrm{r}= \\
0.141\end{array}$ & $\mathrm{~N} / \mathrm{A}$ & $\mathrm{r}=0.01$ & $\mathrm{~N} / \mathrm{A}$ & $\mathrm{N} / \mathrm{A}$ & 0.03 \\
\hline & $\begin{array}{l}\text { 4. Degree of } \\
\text { customer needs } \\
\text { changing in the } \\
\text { market }\end{array}$ & $\begin{array}{l}\mathrm{r}= \\
-0.122\end{array}$ & $\begin{array}{l}\mathrm{r}= \\
0.299\end{array}$ & $\mathrm{r}=0.01$ & $\mathrm{~N} / \mathrm{A}$ & $\begin{array}{c}\mathrm{r}= \\
0.676\end{array}$ & 0.2 \\
\hline
\end{tabular}




\begin{tabular}{|c|c|c|c|c|c|c|c|}
\hline Key factors & Key sub-factors & $\begin{array}{l}\text { Cooper } \\
1979\end{array}$ & $\begin{array}{l}\text { Cooper } \\
\& \\
\text { Kleins- } \\
\text { chmidt } \\
1987\end{array}$ & $\begin{array}{c}\text { Song \& } \\
\text { Parry } \\
1997\end{array}$ & $\begin{array}{l}\text { Song et. } \\
\text { al, } \\
2003^{*}\end{array}$ & $\begin{array}{l}\text { Calantone } \\
\text { et. al, } \\
2008^{* *}\end{array}$ & $\begin{array}{l}\text { AHP } \\
\text { scoring }\end{array}$ \\
\hline & $\begin{array}{l}\text { 5. Markets that } \\
\text { have many new } \\
\text { product } \\
\text { introductions }\end{array}$ & $\begin{array}{l}r= \\
-0.024\end{array}$ & $\begin{array}{l}\mathrm{r}= \\
0.296\end{array}$ & $\mathrm{r}=0.01$ & $\mathrm{~N} / \mathrm{A}$ & $\mathrm{N} / \mathrm{A}$ & 0.06 \\
\hline & $\begin{array}{l}\text { 6. Foreign markets } \\
\text { dominant in the } \\
\text { total of the market }\end{array}$ & $\mathrm{N} / \mathrm{A}$ & $\begin{array}{l}\mathrm{r}= \\
0.331\end{array}$ & $\mathrm{r}=0.01$ & $\mathrm{~N} / \mathrm{A}$ & $\mathrm{N} / \mathrm{A}$ & 0.07 \\
\hline $\begin{array}{l}\text { Firm's } \\
\text { resources } \\
\text { and }\end{array}$ & $\begin{array}{l}1 \text { Sales force or } \\
\text { resources and skills } \\
\text { distribution }\end{array}$ & $\begin{array}{l}\mathrm{r}= \\
0.374\end{array}$ & $\begin{array}{l}\mathrm{r}= \\
0.301\end{array}$ & $\mathrm{r}=0.16$ & $\mathrm{~N} / \mathrm{A}$ & $\mathrm{N} / \mathrm{A}$ & 0.17 \\
\hline \multirow{9}{*}{$\begin{array}{l}\text { Product- } \\
\text { organisatio- } \\
\text { nal fit } \\
(\text { Mean }= \\
0.2)\end{array}$} & $\begin{array}{l}\text { 2. Personnel and } \\
\text { skills of the } \\
\text { marketing research } \\
\text { division }\end{array}$ & $\begin{array}{l}\mathrm{r}= \\
0.372\end{array}$ & $\begin{array}{l}\mathrm{r}= \\
0.332\end{array}$ & $\mathrm{r}=0.16$ & $\mathrm{~N} / \mathrm{A}$ & $\mathrm{N} / \mathrm{A}$ & 0.17 \\
\hline & $\begin{array}{l}\text { 3. Technical skill of } \\
\text { management }\end{array}$ & $\begin{array}{l}\mathrm{r}= \\
0.316\end{array}$ & $\begin{array}{l}r= \\
0.466\end{array}$ & $\mathrm{~N} / \mathrm{A}$ & $\mathrm{r}=0.47$ & $\mathrm{~N} / \mathrm{A}$ & 0.26 \\
\hline & $\begin{array}{l}\text { 4. Marketing skill of } \\
\text { management }\end{array}$ & $\begin{array}{l}\mathrm{r}= \\
0.316\end{array}$ & $\begin{array}{l}\mathrm{r}= \\
0.466\end{array}$ & $\mathrm{~N} / \mathrm{A}$ & $\begin{array}{c}r= \\
0.525\end{array}$ & $\mathrm{~N} / \mathrm{A}$ & 0.27 \\
\hline & $\begin{array}{l}\text { 5. Managerial skill } \\
\text { of management }\end{array}$ & $\begin{array}{l}\mathrm{r}= \\
0.316\end{array}$ & $\begin{array}{l}\mathrm{r}= \\
0.466\end{array}$ & $\mathrm{~N} / \mathrm{A}$ & $\mathrm{r}=0.48$ & $\mathrm{~N} / \mathrm{A}$ & 0.26 \\
\hline & $\begin{array}{l}\text { 6. Cross-functional } \\
\text { integration }\end{array}$ & $\mathrm{N} / \mathrm{A}$ & $\mathrm{N} / \mathrm{A}$ & $\mathrm{r}=0.63$ & $\begin{array}{c}r= \\
0.783\end{array}$ & $\mathrm{~N} / \mathrm{A}$ & 0.31 \\
\hline & $\begin{array}{l}\text { 7. Resources and } \\
\text { skills of the } \\
\text { promotion and } \\
\text { advertisement } \\
\text { division }\end{array}$ & $\begin{array}{l}\mathrm{r}= \\
0.305\end{array}$ & $\mathrm{~N} / \mathrm{A}$ & $\mathrm{r}=0.16$ & $\mathrm{~N} / \mathrm{A}$ & $\mathrm{N} / \mathrm{A}$ & 0.09 \\
\hline & $\begin{array}{l}\text { 8. Resource and } \\
\text { skills of firm's R\&D } \\
\text { and product } \\
\text { development }\end{array}$ & $\begin{array}{l}\mathrm{r}= \\
0.142\end{array}$ & $\begin{array}{l}\mathrm{r}= \\
0.387\end{array}$ & $\mathrm{r}=0.27$ & $\mathrm{~N} / \mathrm{A}$ & $\mathrm{N} / \mathrm{A}$ & 0.16 \\
\hline & $\begin{array}{l}\text { 9. Firm's } \\
\text { engineering skills } \\
\text { and resources }\end{array}$ & $\begin{array}{l}\mathrm{r}= \\
0.213\end{array}$ & $\begin{array}{l}\mathrm{r}= \\
0.329\end{array}$ & $\mathrm{r}=0.27$ & $\mathrm{~N} / \mathrm{A}$ & $\mathrm{N} / \mathrm{A}$ & 0.16 \\
\hline & $\begin{array}{l}\text { 10. Firm's costumer } \\
\text { services skills and } \\
\text { resources }\end{array}$ & $\mathrm{N} / \mathrm{A}$ & $\begin{array}{l}\mathrm{r}= \\
0.256\end{array}$ & $\mathrm{~N} / \mathrm{A}$ & $\mathrm{N} / \mathrm{A}$ & $\mathrm{N} / \mathrm{A}$ & 0.05 \\
\hline
\end{tabular}




\begin{tabular}{|c|c|c|c|c|c|c|c|}
\hline Key factors & Key sub-factors & $\begin{array}{l}\text { Cooper } \\
1979\end{array}$ & $\begin{array}{l}\text { Cooper } \\
\& \\
\text { Kleins- } \\
\text { chmidt } \\
1987\end{array}$ & $\begin{array}{c}\text { Song \& } \\
\text { Parry } \\
1997\end{array}$ & $\begin{array}{l}\text { Song et. } \\
\text { al, } \\
2003^{*}\end{array}$ & $\begin{array}{l}\text { Calantone } \\
\text { et. al, } \\
2008^{* *}\end{array}$ & $\begin{array}{l}\text { AHP } \\
\text { scoring }\end{array}$ \\
\hline & $\begin{array}{l}\text { 11. Rigid reporting } \\
\text { style }\end{array}$ & $\mathrm{N} / \mathrm{A}$ & $\mathrm{N} / \mathrm{A}$ & $\mathrm{N} / \mathrm{A}$ & $\mathrm{N} / \mathrm{A}$ & $\begin{array}{c}r= \\
0.756\end{array}$ & 0.13 \\
\hline & $\begin{array}{l}\text { 12. Flexible } \\
\text { reporting style }\end{array}$ & $\mathrm{N} / \mathrm{A}$ & $\mathrm{N} / \mathrm{A}$ & $\mathrm{N} / \mathrm{A}$ & $\mathrm{N} / \mathrm{A}$ & $\begin{array}{c}r= \\
0.756\end{array}$ & 0.13 \\
\hline & $\begin{array}{l}\text { 13. Formal } \\
\text { managerial style }\end{array}$ & $\mathrm{N} / \mathrm{A}$ & $\mathrm{N} / \mathrm{A}$ & $\mathrm{N} / \mathrm{A}$ & $\begin{array}{c}\mathrm{r}= \\
0.395\end{array}$ & $\begin{array}{c}r= \\
0.726\end{array}$ & 0.21 \\
\hline & $\begin{array}{l}\text { 14. Participative } \\
\text { managerial style }\end{array}$ & $\mathrm{N} / \mathrm{A}$ & $\mathrm{N} / \mathrm{A}$ & $\mathrm{N} / \mathrm{A}$ & $\mathrm{r}=0.5$ & $\begin{array}{c}\mathrm{r}= \\
0.726\end{array}$ & 0.23 \\
\hline & $\begin{array}{l}\text { 15. Managers were } \\
\text { motivating }\end{array}$ & $\mathrm{N} / \mathrm{A}$ & $\mathrm{N} / \mathrm{A}$ & $\mathrm{N} / \mathrm{A}$ & $\mathrm{r}=0.64$ & $\mathrm{~N} / \mathrm{A}$ & 0.14 \\
\hline & $\begin{array}{l}\text { 16. Formal } \\
\text { procedures }\end{array}$ & $\mathrm{N} / \mathrm{A}$ & $\mathrm{N} / \mathrm{A}$ & $\mathrm{N} / \mathrm{A}$ & $\mathrm{N} / \mathrm{A}$ & $\mathrm{r}=0.694$ & 0.12 \\
\hline & $\begin{array}{l}\text { 17. Informal } \\
\text { procedures }\end{array}$ & $\mathrm{N} / \mathrm{A}$ & $\mathrm{N} / \mathrm{A}$ & $\mathrm{N} / \mathrm{A}$ & $\mathrm{N} / \mathrm{A}$ & $\mathrm{r}=0.694$ & 0.12 \\
\hline & $\begin{array}{l}\text { 18. Few people had } \\
\text { authority on the } \\
\text { project }\end{array}$ & $\mathrm{N} / \mathrm{A}$ & $\mathrm{N} / \mathrm{A}$ & $\mathrm{N} / \mathrm{A}$ & $\mathrm{r}=0.4$ & $\mathrm{~N} / \mathrm{A}$ & 0.09 \\
\hline & $\begin{array}{l}\text { 19. Highly } \\
\text { supportive top-level } \\
\text { management }\end{array}$ & $\mathrm{N} / \mathrm{A}$ & $\mathrm{N} / \mathrm{A}$ & $\mathrm{N} / \mathrm{A}$ & $\begin{array}{c}r= \\
0.725\end{array}$ & $\mathrm{~N} / \mathrm{A}$ & 0.16 \\
\hline & $\begin{array}{l}\text { 20. Top-level } \\
\text { management } \\
\text { involvement }\end{array}$ & $\mathrm{N} / \mathrm{A}$ & $\mathrm{N} / \mathrm{A}$ & $\mathrm{N} / \mathrm{A}$ & $\mathrm{r}=0.7$ & $\mathrm{~N} / \mathrm{A}$ & 0.15 \\
\hline \multirow{4}{*}{$\begin{array}{l}\text { Nature of } \\
\text { the venture } \\
(\text { mean }= \\
0.09)\end{array}$} & $\begin{array}{l}\text { 1. Innovativeness of } \\
\text { the product to the } \\
\text { market }\end{array}$ & $\begin{array}{l}\mathrm{r}= \\
0.199\end{array}$ & $\mathrm{~N} / \mathrm{A}$ & $\mathrm{N} / \mathrm{A}$ & $\mathrm{N} / \mathrm{A}$ & $\begin{array}{c}\mathrm{r}= \\
0.730\end{array}$ & 0.17 \\
\hline & $\begin{array}{l}\text { 2. Newness of the } \\
\text { customer need } \\
\text { served to the firm }\end{array}$ & $\begin{array}{l}r= \\
-0.169\end{array}$ & $\mathrm{~N} / \mathrm{A}$ & $\mathrm{N} / \mathrm{A}$ & $\mathrm{N} / \mathrm{A}$ & $\mathrm{N} / \mathrm{A}$ & -0.03 \\
\hline & $\begin{array}{l}\text { 3. Newness of the } \\
\text { product to the firm }\end{array}$ & $\begin{array}{l}r= \\
-0.163\end{array}$ & $\mathrm{~N} / \mathrm{A}$ & $\mathrm{N} / \mathrm{A}$ & $\mathrm{N} / \mathrm{A}$ & $\begin{array}{c}r= \\
0.730\end{array}$ & 0.1 \\
\hline & $\begin{array}{l}\text { 4. Mechanical and } \\
\text { technical } \\
\text { complexity of the } \\
\text { new product }\end{array}$ & $\begin{array}{l}\mathrm{r}= \\
0.032\end{array}$ & $\mathrm{~N} / \mathrm{A}$ & $\mathrm{N} / \mathrm{A}$ & $\mathrm{N} / \mathrm{A}$ & $\begin{array}{c}\mathrm{r}= \\
0.734\end{array}$ & 0.13 \\
\hline
\end{tabular}




\begin{tabular}{|c|c|c|c|c|c|c|c|}
\hline Key factors & Key sub-factors & $\begin{array}{l}\text { Cooper } \\
1979\end{array}$ & $\begin{array}{l}\text { Cooper } \\
\& \\
\text { Kleins- } \\
\text { chmidt } \\
1987\end{array}$ & $\begin{array}{c}\text { Song \& } \\
\text { Parry } \\
1997\end{array}$ & $\begin{array}{l}\text { Song et. } \\
\text { al, } \\
2003^{*}\end{array}$ & $\begin{array}{l}\text { Calantone } \\
\text { et. al, } \\
2008^{* *}\end{array}$ & $\begin{array}{l}\text { AHP } \\
\text { scoring }\end{array}$ \\
\hline \multirow[t]{10}{*}{$\begin{array}{l}\text { Commercial } \\
\text { entity } \\
(\text { mean }= \\
0.16)\end{array}$} & $\begin{array}{l}\text { 1. Degree of which } \\
\text { the product met } \\
\text { consumer needs } \\
\text { better that the } \\
\text { competition }\end{array}$ & $\begin{array}{l}\mathrm{r}= \\
0.492\end{array}$ & $\begin{array}{l}\mathrm{r}=0 \\
556\end{array}$ & $\mathrm{r}=0.48$ & $\mathrm{~N} / \mathrm{A}$ & $\mathrm{N} / \mathrm{A}$ & 0.31 \\
\hline & $\begin{array}{l}\text { 2. Quality of the } \\
\text { product }\end{array}$ & $\begin{array}{l}r= \\
0.416\end{array}$ & $\begin{array}{l}\mathrm{r}= \\
0.441\end{array}$ & $\mathrm{r}=0.48$ & $\mathrm{~N} / \mathrm{A}$ & $\mathrm{N} / \mathrm{A}$ & 0.27 \\
\hline & $\begin{array}{l}3 . \text { Sales forces } \\
\text { accurate targeting } \\
\text { the market }\end{array}$ & $\begin{array}{l}\mathrm{r}= \\
0.410\end{array}$ & $\begin{array}{l}\mathrm{r}= \\
0.378\end{array}$ & $\mathrm{~N} / \mathrm{A}$ & $\mathrm{N} / \mathrm{A}$ & $\mathrm{N} / \mathrm{A}$ & 0.15 \\
\hline & $\begin{array}{l}\text { 4. Effectiveness of } \\
\text { the advertisement } \\
\text { and promotion } \\
\text { efforts }\end{array}$ & $\begin{array}{l}\mathrm{r}= \\
0.233\end{array}$ & $\mathrm{~N} / \mathrm{A}$ & $\mathrm{N} / \mathrm{A}$ & $\mathrm{N} / \mathrm{A}$ & $\mathrm{N} / \mathrm{A}$ & 0.05 \\
\hline & $\begin{array}{l}\text { 5. New product } \\
\text { that offered unique } \\
\text { benefits not found } \\
\text { in the competition }\end{array}$ & $\begin{array}{l}\mathrm{r}= \\
0.300\end{array}$ & $\begin{array}{l}\mathrm{r}= \\
0.342\end{array}$ & $\mathrm{r}=0.48$ & $\mathrm{~N} / \mathrm{A}$ & $\mathrm{N} / \mathrm{A}$ & 0.23 \\
\hline & $\begin{array}{l}\text { 6. New product } \\
\text { solved customer's } \\
\text { problem better than } \\
\text { competitors }\end{array}$ & $\mathrm{N} / \mathrm{A}$ & $\begin{array}{l}\mathrm{r}= \\
0.318\end{array}$ & $\mathrm{~N} / \mathrm{A}$ & $\mathrm{N} / \mathrm{A}$ & $\mathrm{N} / \mathrm{A}$ & 0.06 \\
\hline & $\begin{array}{l}\text { 7. Product reduced } \\
\text { customer cost }\end{array}$ & $\begin{array}{l}\mathrm{r}= \\
0.378\end{array}$ & $\begin{array}{l}\mathrm{r}= \\
0.258\end{array}$ & $\mathrm{~N} / \mathrm{A}$ & $\mathrm{N} / \mathrm{A}$ & $\mathrm{N} / \mathrm{A}$ & 0.12 \\
\hline & $\begin{array}{l}\text { 8. Product that } \\
\text { permitted } \\
\text { customers to } \\
\text { performed unique } \\
\text { tasks }\end{array}$ & $\begin{array}{l}\mathrm{r}= \\
0.238\end{array}$ & $\begin{array}{l}r= \\
0.304\end{array}$ & $\mathrm{r}=0.48$ & $\mathrm{~N} / \mathrm{A}$ & $\mathrm{N} / \mathrm{A}$ & 0.21 \\
\hline & $\begin{array}{l}\text { 9. New product } \\
\text { that used advanced } \\
\text { technology }\end{array}$ & $\mathrm{N} / \mathrm{A}$ & $\begin{array}{l}\mathrm{r}= \\
0.249\end{array}$ & $\mathrm{~N} / \mathrm{A}$ & $\mathrm{N} / \mathrm{A}$ & $\mathrm{N} / \mathrm{A}$ & 0.05 \\
\hline & $\begin{array}{l}\text { 10. Introduction of } \\
\text { a superior product }\end{array}$ & $\mathrm{N} / \mathrm{A}$ & $\begin{array}{l}\mathrm{r}= \\
0.421\end{array}$ & $\mathrm{r}=0.48$ & $\mathrm{~N} / \mathrm{A}$ & $\mathrm{N} / \mathrm{A}$ & 0.19 \\
\hline $\begin{array}{l}\text { Proficiencies } \\
\text { of processes }\end{array}$ & $\begin{array}{l}\text { 1. Exploratory } \\
\text { stage }\end{array}$ & $\mathrm{N} / \mathrm{A}$ & $\mathrm{N} / \mathrm{A}$ & $\mathrm{N} / \mathrm{A}$ & $\begin{array}{c}\mathrm{r}= \\
0.625\end{array}$ & $\mathrm{~N} / \mathrm{A}$ & 0.14 \\
\hline $\begin{array}{l}(\text { mean }= \\
0.18)\end{array}$ & $\begin{array}{l}\text { 2. Concept } \\
\text { development }\end{array}$ & $\mathrm{N} / \mathrm{A}$ & $\mathrm{N} / \mathrm{A}$ & $\mathrm{N} / \mathrm{A}$ & $\mathrm{r}=0.68$ & $\mathrm{~N} / \mathrm{A}$ & 0.15 \\
\hline
\end{tabular}




\begin{tabular}{|c|c|c|c|c|c|c|c|}
\hline Key factors & Key sub-factors & $\begin{array}{l}\text { Cooper } \\
1979\end{array}$ & $\begin{array}{l}\text { Cooper } \\
\& \\
\text { Kleins- } \\
\text { chmidt } \\
1987\end{array}$ & $\begin{array}{c}\text { Song \& } \\
\text { Parry } \\
1997\end{array}$ & $\begin{array}{l}\text { Song et. } \\
\text { al, } \\
2003^{*}\end{array}$ & $\begin{array}{l}\text { Calantone } \\
\text { et. al, } \\
2008^{* *}\end{array}$ & $\begin{array}{l}\text { AHP } \\
\text { scoring }\end{array}$ \\
\hline & $\begin{array}{l}\text { 3. Preliminary } \\
\text { market assessment }\end{array}$ & $\begin{array}{l}\mathrm{r}= \\
0.328\end{array}$ & $\mathrm{~N} / \mathrm{A}$ & $\mathrm{r}=0.11$ & $\begin{array}{c}\mathrm{r}= \\
0.775\end{array}$ & $\mathrm{~N} / \mathrm{A}$ & 0.26 \\
\hline & 4. Market research & $\begin{array}{l}\mathrm{r}= \\
0.342\end{array}$ & $\mathrm{~N} / \mathrm{A}$ & $\mathrm{r}=0.11$ & $\begin{array}{c}\mathrm{r}= \\
0.775 \\
\end{array}$ & $\begin{array}{c}r= \\
0.602 \\
\end{array}$ & 0.36 \\
\hline & $\begin{array}{l}\text { 5. Prototype testing } \\
\text { with the consumer }\end{array}$ & $\begin{array}{l}\mathrm{r}= \\
0.415\end{array}$ & $\mathrm{~N} / \mathrm{A}$ & $\mathrm{r}=0.11$ & $\mathrm{r}=0.76$ & $\mathrm{~N} / \mathrm{A}$ & 0.27 \\
\hline & $\begin{array}{l}\text { 6. Test marketing } \\
\text { trial selling }\end{array}$ & $\begin{array}{l}r= \\
0.407\end{array}$ & $\mathrm{~N} / \mathrm{A}$ & $\mathrm{r}=0.11$ & $\mathrm{~N} / \mathrm{A}$ & $\mathrm{N} / \mathrm{A}$ & 0.1 \\
\hline & 7. Market lunch & $\begin{array}{l}\mathrm{r}= \\
0.517\end{array}$ & $\mathrm{~N} / \mathrm{A}$ & $\mathrm{r}=0.11$ & $\mathrm{~N} / \mathrm{A}$ & $\mathrm{N} / \mathrm{A}$ & 0.13 \\
\hline & 8. Initial screening & $\begin{array}{l}\mathrm{r}= \\
0.370\end{array}$ & $\mathrm{~N} / \mathrm{A}$ & $\mathrm{N} / \mathrm{A}$ & $\mathrm{N} / \mathrm{A}$ & $\mathrm{N} / \mathrm{A}$ & 0.07 \\
\hline & $\begin{array}{l}\text { 9. Financial } \\
\text { analysis }\end{array}$ & $\begin{array}{l}\mathrm{r}= \\
0.309\end{array}$ & $\mathrm{~N} / \mathrm{A}$ & $\mathrm{N} / \mathrm{A}$ & $\mathrm{N} / \mathrm{A}$ & $\mathrm{N} / \mathrm{A}$ & 0.06 \\
\hline & 10. Trail production & $\begin{array}{l}\mathrm{r}= \\
0.267\end{array}$ & $\mathrm{~N} / \mathrm{A}$ & $\mathrm{r}=0.43$ & $\mathrm{~N} / \mathrm{A}$ & $\mathrm{N} / \mathrm{A}$ & 0.15 \\
\hline & $\begin{array}{l}\text { 11. Preliminary } \\
\text { technical assessment }\end{array}$ & $\begin{array}{l}\mathrm{r}= \\
0.282\end{array}$ & $\mathrm{~N} / \mathrm{A}$ & $\mathrm{r}=0.43$ & $\mathrm{~N} / \mathrm{A}$ & $\mathrm{N} / \mathrm{A}$ & 0.15 \\
\hline & $\begin{array}{l}\text { 12. Product } \\
\text { development }\end{array}$ & $\begin{array}{l}r= \\
0.394\end{array}$ & $\mathrm{~N} / \mathrm{A}$ & $\mathrm{r}=0.43$ & $\mathrm{~N} / \mathrm{A}$ & $\mathrm{N} / \mathrm{A}$ & 0.17 \\
\hline & $\begin{array}{l}\text { 13. Prototype } \\
\text { development }\end{array}$ & $\mathrm{N} / \mathrm{A}$ & $\mathrm{N} / \mathrm{A}$ & $\mathrm{N} / \mathrm{A}$ & $\begin{array}{c}\mathrm{r}= \\
0.735\end{array}$ & $\mathrm{~N} / \mathrm{A}$ & 0.16 \\
\hline & $\begin{array}{l}\text { 14. Prototyping and } \\
\text { testing in-house }\end{array}$ & $\begin{array}{l}\mathrm{r}= \\
0.325\end{array}$ & $\mathrm{~N} / \mathrm{A}$ & $\mathrm{r}=0.43$ & $\mathrm{r}=0.76$ & $\mathrm{~N} / \mathrm{A}$ & 0.32 \\
\hline & $\begin{array}{l}\text { 15. Full production } \\
\text { start-up }\end{array}$ & $\begin{array}{l}\mathrm{r}= \\
0.394\end{array}$ & $\mathrm{~N} / \mathrm{A}$ & $\mathrm{r}=0.43$ & $\begin{array}{c}r= \\
0.655\end{array}$ & $\mathrm{~N} / \mathrm{A}$ & 0.31 \\
\hline & $\begin{array}{l}\text { 16. Technical } \\
\text { service }\end{array}$ & $\mathrm{N} / \mathrm{A}$ & $\mathrm{N} / \mathrm{A}$ & $\mathrm{N} / \mathrm{A}$ & $\mathrm{r}=0.61$ & $\mathrm{~N} / \mathrm{A}$ & 0.13 \\
\hline \multirow{2}{*}{$\begin{array}{l}\text { Information } \\
\text { acquired } \\
(\text { mean }= \\
0.21)\end{array}$} & $\begin{array}{l}\text { 1.Knowledge of } \\
\text { consumer price } \\
\text { sensitivity }\end{array}$ & $\begin{array}{c}r= \\
0.391\end{array}$ & $\mathrm{~N} / \mathrm{A}$ & $\mathrm{r}=0.45$ & $\mathrm{~N} / \mathrm{A}$ & $\mathrm{N} / \mathrm{A}$ & 0.17 \\
\hline & $\begin{array}{l}\text { 2.Understanding } \\
\text { buyer's behaviour }\end{array}$ & $\begin{array}{c}r= \\
0.391\end{array}$ & $\mathrm{~N} / \mathrm{A}$ & $\mathrm{N} / \mathrm{A}$ & $\mathrm{N} / \mathrm{A}$ & $\mathrm{N} / \mathrm{A}$ & 0.08 \\
\hline
\end{tabular}




\begin{tabular}{|c|c|c|c|c|c|c|c|}
\hline Key factors & Key sub-factors & $\begin{array}{l}\text { Cooper } \\
1979\end{array}$ & $\begin{array}{l}\text { Cooper } \\
\& \\
\text { Kleins- } \\
\text { chmidt } \\
1987\end{array}$ & $\begin{array}{c}\text { Song \& } \\
\text { Parry } \\
1997\end{array}$ & $\begin{array}{l}\text { Song et. } \\
\text { al, } \\
2003^{*}\end{array}$ & $\begin{array}{l}\text { Calantone } \\
\text { et. al, } \\
2008^{* *}\end{array}$ & $\begin{array}{c}\text { AHP } \\
\text { scoring }\end{array}$ \\
\hline & $\begin{array}{l}\text { 3.Knowledge of } \\
\text { consumer needs, } \\
\text { wants and technical } \\
\text { aspects needed for } \\
\text { the new product }\end{array}$ & $\begin{array}{c}r= \\
0.362\end{array}$ & $\begin{array}{c}r= \\
0.590\end{array}$ & $\mathrm{r}=0.45$ & $\mathrm{~N} / \mathrm{A}$ & $\begin{array}{c}r= \\
0.783\end{array}$ & 0.42 \\
\hline & $\begin{array}{l}\text { 4.Knowledge about } \\
\text { the competition } \\
\text { product, strategy, } \\
\text { pricing }\end{array}$ & $\begin{array}{c}r= \\
0.331\end{array}$ & $\mathrm{~N} / \mathrm{A}$ & $\mathrm{r}=0.45$ & $\mathrm{~N} / \mathrm{A}$ & $\begin{array}{c}r= \\
0.753\end{array}$ & 0.29 \\
\hline & $\begin{array}{l}\text { 5.Knowledge about } \\
\text { competitor reaction } \\
\text { after new product } \\
\text { launch }\end{array}$ & $\mathrm{N} / \mathrm{A}$ & $\mathrm{N} / \mathrm{A}$ & $\mathrm{r}=0.45$ & $\mathrm{~N} / \mathrm{A}$ & $\begin{array}{c}r= \\
0.586\end{array}$ & 0.2 \\
\hline & $\begin{array}{l}\text { 6. Market size } \\
\text { knowledge }\end{array}$ & $\begin{array}{c}r= \\
0.284\end{array}$ & $\mathrm{~N} / \mathrm{A}$ & $\mathrm{r}=0.45$ & $\mathrm{~N} / \mathrm{A}$ & $\mathrm{N} / \mathrm{A}$ & 0.15 \\
\hline & $\begin{array}{l}\text { 7.Understanding } \\
\text { new products } \\
\text { technology }\end{array}$ & $\begin{array}{c}r= \\
0.203\end{array}$ & $\mathrm{~N} / \mathrm{A}$ & $\mathrm{N} / \mathrm{A}$ & $\mathrm{N} / \mathrm{A}$ & $\begin{array}{c}r= \\
0.667\end{array}$ & 0.16 \\
\hline \multirow{5}{*}{$\begin{array}{l}\text { Project } \\
\text { definition } \\
(\text { mean }= \\
0.11)\end{array}$} & 1.Project concept & $\mathrm{N} / \mathrm{A}$ & $\begin{array}{c}r= \\
0.420\end{array}$ & $\mathrm{~N} / \mathrm{A}$ & $\mathrm{N} / \mathrm{A}$ & $\mathrm{N} / \mathrm{A}$ & 0.08 \\
\hline & $\begin{array}{l}\text { 2.Project } \\
\text { requirements and } \\
\text { specifications }\end{array}$ & $\mathrm{N} / \mathrm{A}$ & $\begin{array}{c}\mathrm{r}= \\
0.329\end{array}$ & $\mathrm{~N} / \mathrm{A}$ & $\mathrm{N} / \mathrm{A}$ & $\mathrm{N} / \mathrm{A}$ & 0.06 \\
\hline & $\begin{array}{l}\text { 3.Project detailed } \\
\text { planning and } \\
\text { control }\end{array}$ & $\mathrm{N} / \mathrm{A}$ & $\mathrm{N} / \mathrm{A}$ & $\mathrm{N} / \mathrm{A}$ & $\begin{array}{c}r= \\
0.625\end{array}$ & $\mathrm{~N} / \mathrm{A}$ & 0.14 \\
\hline & $\begin{array}{l}\text { 4.Participation of } \\
\text { all project team } \\
\text { members in } \\
\text { planning }\end{array}$ & $\mathrm{N} / \mathrm{A}$ & $\mathrm{N} / \mathrm{A}$ & $\mathrm{N} / \mathrm{A}$ & $\mathrm{r}=0.72$ & $\mathrm{~N} / \mathrm{A}$ & 0.16 \\
\hline & $\begin{array}{l}5 . \text { Rigid hierarchical } \\
\text { reporting in } \\
\text { relationship to } \\
\text { planning }\end{array}$ & $\mathrm{N} / \mathrm{A}$ & $\mathrm{N} / \mathrm{A}$ & $\mathrm{N} / \mathrm{A}$ & $\begin{array}{c}r= \\
0.565\end{array}$ & $\mathrm{~N} / \mathrm{A}$ & 0.12 \\
\hline $\begin{array}{l}\text { Firms' } \\
\text { Strategy } \\
(\text { mean= } \\
0.12)\end{array}$ & $\begin{array}{l}\text { 1.Level of } \\
\text { aggressiveness } \\
\text { toward competitors }\end{array}$ & $\mathrm{N} / \mathrm{A}$ & $\mathrm{N} / \mathrm{A}$ & $\mathrm{N} / \mathrm{A}$ & $\mathrm{N} / \mathrm{A}$ & $\begin{array}{c}r= \\
0.666\end{array}$ & 0.12 \\
\hline
\end{tabular}




\begin{tabular}{|c|c|c|c|c|c|c|c|}
\hline Key factors & Key sub-factors & $\begin{array}{l}\text { Cooper } \\
1979\end{array}$ & $\begin{array}{l}\text { Cooper } \\
\& \\
\text { Kleins- } \\
\text { chmidt } \\
1987\end{array}$ & $\begin{array}{c}\text { Song \& } \\
\text { Parry } \\
1997\end{array}$ & $\begin{array}{l}\text { Song et. } \\
\text { al, } \\
2003^{*}\end{array}$ & $\begin{array}{l}\text { Calantone } \\
\text { et. al, } \\
2008^{* *}\end{array}$ & $\begin{array}{l}\text { AHP } \\
\text { scoring }\end{array}$ \\
\hline & $\begin{array}{l}\text { 2.Firm's behaviour } \\
\text { in respect to action }\end{array}$ & $\mathrm{N} / \mathrm{A}$ & $\mathrm{N} / \mathrm{A}$ & $\mathrm{N} / \mathrm{A}$ & $\mathrm{N} / \mathrm{A}$ & $\begin{array}{c}r= \\
0.713\end{array}$ & 0.12 \\
\hline & $\begin{array}{l}\text { 3.Degree of risk } \\
\text { taking in respect to } \\
\text { decision-making }\end{array}$ & $\mathrm{N} / \mathrm{A}$ & $\mathrm{N} / \mathrm{A}$ & $\mathrm{N} / \mathrm{A}$ & $\mathrm{N} / \mathrm{A}$ & $\begin{array}{c}r= \\
0.696\end{array}$ & 0.12 \\
\hline
\end{tabular}

Note*: Song et. al, 2003's obtained results for both Korea and Japan separately. The illustrations exhibit the mean correlation coefficients of both countries. Note**: all correlation coefficients were significant at 0.01. However, Calantone et. al, 2008's results were significant at 0.05. Therefore, their results were scaled down to be compatible.

\section{Findings and discussion}

"Information acquired" is indicated as the most important key factor from the results obtained from the AHP scoring, with a mean AHP score of 0.21 (Table 1). It is composed of all the new information gathered in every stage of the NPD processes, and needs to be shared with every new product project team member and departments for the successful development of the new product. At each stage of the new product process new information is acquired, which Cooper (1979), referred to as a "controllable" factor. This factor is essential as the information and knowledge acquired can have a significant influence on the success or failure of the new product project.

The "Information acquired" factor has seven sub-factors. The most influential sub-factor being "Knowledge of consumer needs, wants and technical aspects needed for the new product" (AHP score 0.42 ). "Knowledge about the competition product, strategy, pricing" and "Knowledge about competitor reaction after new product launch" sub-factors are considered moderately influential having AHP scores of 0.29 and 0.20 respectively.

A "Firm's resources and organisation-product fit" (mean AHP score 0.2) is the second key factor identified from the results in table 1 and the most influential sub-factor of this aspect was "Crossfunctional integration" (AHP score 0.31). The analysis summarised in table 1 also indicates that the "Firm's resources and organisation-product fit" factor is made up of organisationalproduct fits, namely technical and marketing synergies and the skills and resources of different departments. The analysis also indicates that managerial and operational styles are also elements of organisational resources.

According to Cooper and Kleinschmidt (1987) the synergy or organisational fit is also an aspect that influences the financial success of the NPD project and they argue that organisations that produce new products that fit with the organisation's resources, skills and knowledge will 
give higher chances of financial success. Synergy in areas such as; resources and skills, R\&D and product development, marketing research, engineering, salesforce and distribution, consumer services, production and advertisement impact the financial success of NPD projects. Furthermore, these synergy areas could be used as a screening method for the evaluation of a new product project. (Cooper \& Kleinschmidt, 1987)

Song and Parry (1997) found that organisations' technical and marketing skills and sources (synergy) were essential for success in NPD projects. They also recognised that for new product projects to be successful, team leaders and new product project managers need to stay close to the knowledge, resources and skills they have obtained in the past and further stay close to their previous product-lines.

Moreover, according to Song et. al, (2003) the relationships of project management, structural and outcome dimensions have on the survival of NPD projects and the underlying key factor of "Cross-functional integration" impacts positively on the survival of a new product project. Further, improving cross-functional integration and planning the NPD project will result in an improvement in project management practices and project performance.

The third key factor that this paper reviewed was the "Proficiency of processes" (mean AHP score 0.18) which involves the stages of the NPD process itself from idea generation to launch within the target market. The most influential sub-factor of this is "Market research" (AHP score 0.36). Other sub-factors identified as influential are "Prototyping and testing in-house" and "Full production start-up" (AHP scores 0.32 and 0.31 respectively). Further, this key factor is among the few parameters that was identified by each author examined, as consistently influential and reliable.

The fourth key factor examined by the summary table was "Commercial entity" (AHP score 0.16 ) which is considered as representing the characteristics or competitive advantage of the new product.

This key factor has ten sub-factors the most influential being "Degree of which the product met consumer needs better that the competition" (AHP score 0.31), with other influential subfactors being "Quality of the product", "New product that offered unique benefits not found in the competition" and "Introduction of a superior product".

According to Cooper (1979), the "Commercial entity" is the result of the new product process and entails different goal-based tasks and data collection roles, such as, idea creation, marketing research, production and roll out to the market, all of which influence the outcome of the new product project. Hence, the method of carrying out these activities, the quality and method of data collection impacts on the entity of the finished product.

Furthermore, the products that were successful were the ones that gained a significant amount of market share both domestically and abroad (Cooper and Kleinschmidt, 1987). The factors that affected this performance measure had strong ties to the advantages the new product offered, and its characteristics, for example: product superiority, product quality, benefits given to the consumer based on new product features, a product that fulfils the consumers' needs and the use of advance technology.

The fifth key factor according to the summary table was "Firm's strategy" (AHP score 0.12), 
which had three main sub-factors namely "Level of aggressiveness toward competitors", "Firm's behaviour in respect to action" and "Degree of risk-taking in respect to decision-making" (all with AHP score 0.12). Calantone et. al, (2008) is the only work that regarded strategy as a separate factor and noted that a proactive strategy coupled with an organic organisational structure influence marketing intelligence and innovativeness and have positive influences on the outcome of the NPD project.

According to Cooper and Kleinschmidt (1987), the success of the new project has three independent dimensions, financial, market, and opportunity, which are measured differently and have different influencing factors. They encourage managers to consider each dimension when evaluating the success or failure of the new product project and establishing the firm's strategy regarding the new product. Therefore, this echoes the view that firms need to adopt a relevant strategy to achieve the intended outcome for the new product.

Song and Parry (1997) confirm the impact of marketing and competitive intelligence, market activity proficiency, and product competitive advantage, on the project management aspect of the new product project programme, however, they observed that in Japanese firms the market activity proficiency, which directly affects new product competitive advantage and new product success positively, is less important. This, they say, is due to Japanese organisations' value of technical proficiency over marketing activities and suggest that is due to the culture and societal differences between Japan and the west. It could be therefore be said that strategies differ between countries based on the needs and norms of the organisation and culture. (Song \& Parry 1997)

According to table 1, the sixth key factor is "Project definition" (mean AHP score 0.11), which has five sub-factors, the most influential being the "Participation of all project team members in planning" (AHP score 0.16). This factor entails the planning, protocols, requirements and specifications of the NPD project.

Song et. Al, (2003) examined three sub-factors of project definition, which were identified as planning, which contributes to the survival of the new product project within the market; detailed planning, with all team members contributing to the planning process; and autonomy and flexibility of the project teams. Detailed planning could increase the efficiency of the development process which in turn could impact the efficiency and overall performance of the new product within the market. Top-level mangers and project leaders need to ensure the participation of all members in the planning stage of the NPD project, which leads to each team members being responsible for the new project. (Song et. Al, 2003)

The AHP scores shown in table 1 indicate that two factors were not influential, namely "Market environment" and "Nature of the venture" both with a mean AHP score of 0.09.

"Market environment" is a characteristic of the market and has six sub-factors two of which had a moderate influence. These were "Degree of customer needs changing in the market" (AHP score $0.20)$ and "Customer need of the specific product" (AHP score 0.13).

"Market environment" is noteworthy as Cooper (1979) found that it had a minimal impact on the outcome of the new product, but that market information and marketing activities were important due to the impact that they have on the outcome of the product. These results accept 
that the nature of the market place, which the new product is targeted at, has a negligible effect, and conflicts with the emphasis put on the nature of the market within most qualitative screening methods.

Cooper's findings were supported by Song and Parry (1997), who noted that the development of new products was based on technologies that the consumer will value. This could be assisted by close interaction and better relations with the consumers in the markets that the new product is intended for. (Song \& Parry 1997)

Likewise, according to Calantone et. al, (2006), the integration of marketing intelligence within other steps of the NPD process is essential for success. Hence, detailed, planned, and scientific marketing research is key for NPD project success.

Cooper and Kleinschmidt (1987), say that the market environment's lack of importance could be due to the market conditions such as size, growth and rate, etc. having little impact on the market share the new product project will obtain for the organisation. For example, larger markets could be attractive at first to the organisation but it could be highly competitive, making niche markets sometimes more appropriate. Moreover, there was more emphasis in their study on companies' foreign markets, as, if the new product had significant success in the foreign markets it more likely have the success in home markets. (Cooper \& Kleinschmidt, 1987)

"Nature of the venture" (mean AHP score 0.09), which represents the characteristics of the new product project is the factor second least likely to be influential and includes four subfactors, none of which have a strong influence. Of these "Innovativeness of the product to the market" and "Mechanical and technical complexity of the new product" (AHP score 0.17 and 0.13 respectively), have the highest score but nevertheless have limited influence.

According to Cooper (1979), "Nature of the venture" has a relatively low impact on the outcome of the new product project. However, he argues that the dilemma that organisations face during new product project development is choosing the best level of product innovativeness, presented by him as the sub-factor "Newness of the product". This is strongly related to the success of the project but the "Degree of product and market newness" to the organisation is highly related to its failure. However, this is not a great anomaly as these sub-factors are not related to each other. Hence, organisations could be able to select and manufacture highly innovative products without obtaining new costumers or the need to shift to a new product category.

Cooper (1979) also says that, models of new product screening usually use the newness aspect as it could be a proxy for product-organisation fit and synergy. However, he found an unexpected result, as the most influential environmental variable is the firm's resource capabilities but that newness to the organisation is not so important. He also found that the three important subfactors of newness, which are technological, production and consumer, are significantly related to the firm's capabilities but, this relation is not robust enough to consider these factors as proxies for each other. Cooper therefore concludes that the newness sub-factor with its variables is not an efficient measure for new product success.

Calantone et. al, (2008), however, examined environmental turbulence, which consists of technological uncertainty and market turbulence. Environmental turbulence has a moderating effect on the relationship that innovativeness and market intelligence have on the outcome of the new 
product project. Hence, during a low-turbulent environment, market intelligence has a positive impact on the successful outcome but during highly turbulent environments the relationship between innovativeness and success is more important (Calantone et.al 2008). These effects can be clearly be seen during 2020 pandemic where previous market intelligence has become flawed in many industries.

\section{The proposed conceptual model}

Based on the results obtained from table 1 and literature, this paper's conceptual model is centred on Figure 1:

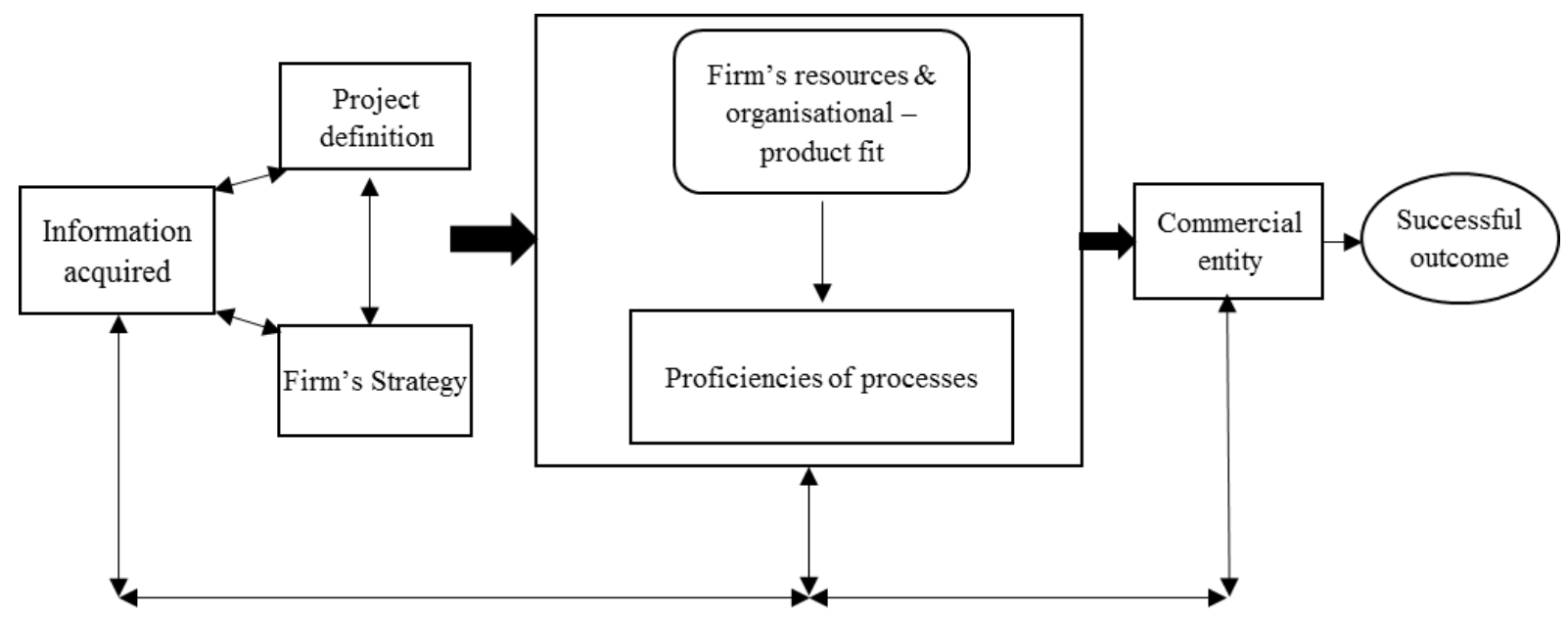

Fig. 1 The proposed Conceptual model structure

According to the proposed conceptual model, the successful outcome of a new product project is directly and significantly influenced by the "Commercial entity" factor. Cooper (1979) suggests that the success or failure of new product projects is directly based on the relationship of a "Commercial entity" with the market; the "Commercial entity" itself is influenced by other factors.

The "Commercial entity" factor is the output of a transformation process, which in this case is represented by the "Proficiency of NPD processes". For the NPD processes to be proficient, the conceptual model concludes that a "Firm's resources \& organisational - product fit" has a direct impact on this factor.

Song and Parry (1997) believe that marketing and technical proficiencies have an indirect link to success. This is because the performance of the actual product is increased by the robust implementation of the technical activities, which leads to the "Competitive advantage" which in this paper is represented as "Commercial entity" as they are defined in the same way in the literature.

Furthermore, the inputs of the transformation process in the model presented in this paper are considered as "Project definition" and "Firm's strategy"; factors which influence each other. 
Finally, the "Information acquired" factor was presumed, in the proposed model, to be at the beginning of the process so that it directly influences the "Project definition" and the "Firm's strategy". Based on the results obtained from this research, the "Information acquired" factor was considered as the underlying key factor, which also indirectly influences the successful outcome of NPD projects. Moreover, this factor is mentioned in relation to other factors in this conceptual model by collecting and sharing information in each stage of the NPD process.

\section{Conclusion}

As a conclusion, this research identified three main sections within the proposed conceptual model, namely the input of the transformation process, the transformation process itself, and the output of the transformation process.

"Commercial entity" which can be interpreted as the output of the transformation process, and defined as the features of the new product, is, based on the results of this paper, the fourth most influential factor on the outcome of NPD with mean AHP score of 0.16.

The second section is the transformation process itself, which generates the "Commercial entity" factor. The transformation process is influenced by a combination of two factors; the "Firm's resources and organisation-product fit" and the "Proficiency of processes" factors.

The "Firm's resources and organisation-product fit" is considered as being the technical and marketing synergies, the skills and resources of different departments as well as managerial management styles. This factor, with, from the results, its twenty sub-factors was considered as the second most influential on the outcome of the NPD with mean AHP score of 0.2.

The "Proficiency of processes" is the second factor within the transformation process, and is defined as the steps, from idea generation to launch, of the NPD process itself. This factor, with its sixteen sub-factors, is considered as the third most influential on the outcome of NPD with a mean AHP score of 0.18 .

The first section, the input of the transformation process, entails the groundwork, planning and strategy-setting, and is reliant on the pool of knowledge and expertise within the organisation. This section is made up of three factors, which are "Information acquired", "Firm's strategy" and "Project definition".

The "Information acquired" factor not only impacts the other factors within the first section of the proposed conceptual model but also indirectly impacts the successful outcome of the NPD process and relates with all sections of the conceptual model by collecting and sharing information which is ultimately held in the pool of knowledge of the organisation. The results show that this factor is the underlying key factor that influences the outcome of NPD with a mean AHP score of 0.21 .

The "Firm's strategy" factor is the strategy of the NPD project and is considered as the fifth most influential factor on the NPD outcome with a mean AHP score of 0.12.

The "Project definition" factor are the planning, protocols, requirements and specifications of the NPD project and is the sixth and least significant factor impacting outcome of the NPD with 
mean AHP scoring of 0.11. This may suggest that the importance of "Project definition", while intuitively thought of as being significant, is influenced by the lack of the need of significant changes in the general procedures from project to project.

It is worth noting that the second and third sections of the proposed conceptual model, the outcome of the transformation process, and the transformation process are recognised in earlier literature. However, the first section, the "Input of the transformation process" result from this study.

\subsection{Future of the research}

This research has captured the work of leading authors on NPD to develop an analytical hierarchy process (AHP) metric for ranking the most significant factors which influence the success of new product projects.

To further enhance and assess the conceptual model, future research will extend the number of publications analysed and include publications by other notable authors.

The factors and sub-factors within the proposed model will be tested in the specific context of an industrial sector in the UK, using a quantitative approach. This may also identify new key factors and sub-factors which could be incorporated in a modified model for the particular industrial context.

\section{References}

Calantone, R., Kandemir, D. and Garcia, R., (2006), "An Exploration of Organisational Factors in New Product Development Success', Journal of Business 83 Industrial Marketing, Vol. 21, No.5, pp. 300-310.

Cooper, R. G., (1979), "Identifying Industrial New Product Success: Project New Prod', Journal of Marketing Management, vol. 8, Issue. 2, pp. 124-135.

Cooper, R.G. and Kleinschmidt, E.J. (1987) "Success factors in product innovation', Journal of Industrial Marketing Management, Vol. 16, No. 3, pp. 215-223.

Droge, C., Calantone, R. and Harmancioglu, N., (2008), "New Product Success: Is It Really Controllable By Managers in Highly Turbulent Environments?', Journal of Product Innovation Management, Vol. 25, pp. 272-286.

Harris, R., and Trainor, M., (2009), "Why Do Some Firms Undertake R\&D Whereas Others Do Not?', Spatial Economics Research Centre (SERC), London School of Economics, Discussion paper 20.

Pisano, G.P., (2012), "Creating an R\&D Strategy', Harvard Business School, Working Paper 12-095.

Song, X.M. and Noh, J., (2006),'Best New Product Development and Management Practices 
in The Korean High-Tech Industry', Journal of Industrial Marketing Management, Vol. 35, pp. 262-278.

Song, X.M. and Parry, M.E., (1997)," The Determinants of Japanese New Product Success', Journal of Marketing Research, Vol. 34, pp. 64-76.

Thieme, J., (2007), "Perspective: The World's Top Innovation Management Scholars and Theirs Social Capital', Journal of Product Innovation Management, Vol. 24, pp. 214-229.

Thieme, J., Song, X.M. and Shin, G., (2003), "Project Management Characteristics and New Product Survival', Journal of Product Innovation Management, Vol. 20, pp. 104-119. 


\section{Biographies}

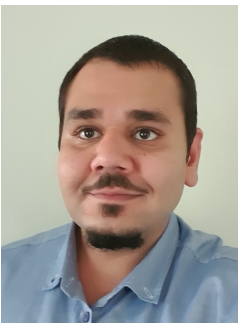

Pouya S. Moghadam. Pouya S. Moghadam is from Tehran, Iran and is a PhD student at the School of Management at Swansea University where he graduated with first-class honours in business management and gained an Msc in Operations and supply-chain management with distinction. His research is on New Product Development with a particular interest in finding methods to streamline the New Product Development process. He is also interested in Lean Operations' and Industry 4.0 research and applications. Since 2019 he has held a position on the Communication Engagement and Community board of Swansea University, creating and maintaining methods of communication between the research community and the executive board of the University. He has presented at a number of conferences including post graduate research conferences at Swansea University and the EuroMA conferences in 2019 and 2020.

CRediT Statement: Conceptualization, Methodology, Validation, Formal analysis, Investigation, Writing - Original Draft, Visualization.

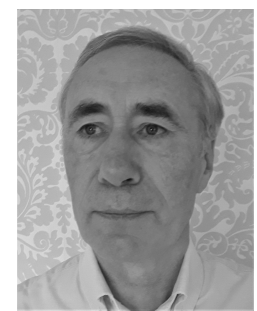

Rhys G. Williams. Rhys G Williams is a Welsh speaker from Llanelli, Wales. He received a BSc in Structural Engineering from Cardiff University, Wales, and following several years employment in Engineering Consultancy and Management Consultancy obtained an MSc in Management Science and Operational Research from Imperial College, London followed by a $\mathrm{PhD}$ in Management Science and Statistics, which was supported by a Science Research Council scholarship. He is a Senior Lecturer at the School of Management of Swansea University where he teaches Management Science and related subjects. He has supervised PhD and MPhil research students in topics ranging from AI applications and learning in product development to investment strategies in Real Options, and has published in academic journals and produced conference papers and book chapters on project evaluation and management as well as other management topics. CRediT Statement: Supervision, Methodology, Validation, Formal analysis, Writing - Original Draft, Writing Review \& Editing, Visualization.

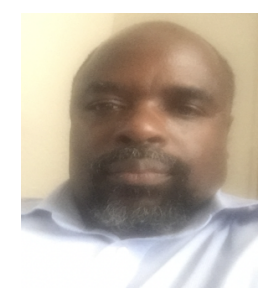

John Mulyata. John Mulyata was born in Zambia. He received the DCMS from Chainama College of Health Sciences, Lusaka, Zambia, and went on to obtain a BSc (Hons), MSc (Econ), MBA and PhD in Business Studies from Swansea University, Swansea, Wales, United Kingdom, in 2016. From 2016 to date a lecturer in Operations and Strategy in the school of management at Swansea University. His research interest is in the area of Procurement and Distribution of logistics, Operations \& Process management and Performance Management in healthcare, Infrastructure, Transport and Health Financing. Since 1986, he has worked in management positions in Healthcare sector, Project Management, and Monitoring and Evaluation. He has conducted consultancy work for healthcare sector in the Ministry of Health in Zambia during the design and implementation of Health Reforms and Health Management Information System (HMIS).

CRediT Statement: Supervision, Validation, Writing - Review 86 Editing.

\section{ISSN 2183-0606}

http://www.open-jim.org

http://creativecommons.org/licenses/by/3.0 